\title{
Decreased phagocytic function in neutrophils and monocytes from peripheral blood in periodontal disease
}

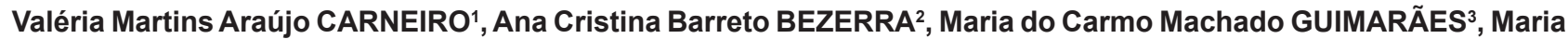 \\ Imaculada MUNIZ-JUNQUEIRA ${ }^{4}$
}

\begin{abstract}
1- DDS, MSc, PhD, Adjunct Professor of Periodontics, Periodontics Division, University of Brasilia, Brasilia, DF, Brazil.
2- DDS, MSc, PhD, Associate Professor of Pediatric Dentistry. Post-Graduation Program Advisor, Health Science Center, University of Brasília, Brasília, DF, Brazil.

3- DDS, MSc, PhD, Adjunct Professor of Periodontics, Periodontics Division, University of Brasilia, Brasilia, DF, Brazil.

4- MD, MSc, PhD, Associate Professor, Laboratory of Cellular Immunology, Faculty of Medicine, University of Brasilia, Brasilia, DF, Brazil.
\end{abstract}

Corresponding address: Valéria Martins Araújo Carneiro - Universidade de Brasília/UnB - Departamento de Odontologia - Campus Universitário Darcy Ribeiro - 70910-900 - Brasília - DF - Brasil - Phone:+55 6133072514 - e-mail: valeriamartins@unb.br

Received: December 02, 2010 - Modification: June 12, 2011 - Accepted: July 08, 2011

\section{ABSTRACT}

\begin{abstract}
Dhagocytosis by neutrophils and monocytes constitutes the main defense mechanism against bacterial challenges in periodontitis. Phagocytosis by neutrophils has already been evaluated, whereas phagocytic function of monocytes has hardly been addressed so far. Objectives: The aim of this study was to assess phagocytosis by neutrophils and monocytes in periodontitis. Material and Methods: The sample included 30 subjects with severe periodontitis and 27 control subjects without periodontal disease. The phagocytic index (PhI) was calculated as the mean number of adhered/ingested Saccharomyces cerevisiae per phagocytozing monocyte or neutrophil multiplied by the percentage of phagocytes involved in phagocytosis. Results: A significant reduction in phagocyte functions was observed in individuals with periodontitis. The median of PhI of neutrophils using nonsensitized $S$. cerevisiae was 3 for the control group, and 1.5 for the periodontitis group $(p=0.01$, Mann-Whitney test). The median of PhI of monocytes with non-sensitized $S$. cerevisiae was 26.13 for the control group, and 13.23 for the periodontitis group $(p=0.03$, Mann Whitney test). The median of PhI of monocytes assessed with sensitized S. cerevisiae was 97.92 for the control group and 60.1 for the periodontitis group $(p=0.005$, t-test). Conclusion: The data demonstrated a reduction in the function of phagocytes, suggesting a decrease in immune defenses in periodontitis.
\end{abstract}

Key words: Periodontitis. Neutrophils. Monocytes. Phagocytosis.

\section{INTRODUCTION}

It has been considered that periodontal infection or periodontitis results from the imbalance between the direct and indirect effects of pathogenic bacteria and the host immune response?. Data support the microbial etiology of periodontal disease and the role played by specific pathogenic species ${ }^{5,25}$. Polymorphonuclear (PMN) neutrophils constitute the first line of defense against these pathogens in the subgingival area. In the presence of plaque, an inflammatory infiltrate is frequently in the gingival tissues, showing mononuclear phagocytes, lymphocytes, and polymorphonuclear leukocytes ${ }^{16,22}$. Additionally, inflammatory response changes the microenvironment of the biofilm and selects for specific organisms ${ }^{28}$. The interaction between lipopolysaccharide (LPS) and these predominant cell types in the inflammatory infiltrate stimulates the production and elevation of prostanoids, mainly prostaglandin $E_{2}$ in the gingival fluid of sites that present attachment loss ${ }^{30}$. In addition to the local effects, the bacterial virulence factors and inflammatory mediators arising from this parasitehost interaction may create and sustain a chronic systemic inflammatory process in the bloodstream ${ }^{17}$. In the most aggressive forms of periodontal disease, the role played by the elevated serum levels of tumor necrosis factor-alpha (TNF-alpha) and interleukin-1 (IL-1) has been considered partly 
responsible for altering the neutrophil function ${ }^{1}$. In addition, new perspectives have indicated that periodontitis occurs as a hyperactive immune/ inflammatory response to the specific bacteria of plaque in predisposed individuals, involving the excessive generation of oxygen radicals and the release of proteases ${ }^{14}$. However, despite this new paradigm that PMN is not "hypofunctional" or "deficient", but "hyperfunctional"14, phagocytosis in this context is not yet elucidated.

Data on phagocytosis by neutrophils from peripheral blood in individuals with periodontitis are controversial. There are reports both of reduction $2,4,12,29$ and increase of phagocytosis by neutrophils ${ }^{13,21}$. Studies addressing the phagocytosis by monocytes in these patients are still scarce. Therefore, it still has to be determined whether phagocytosis by monocytes in individuals with periodontitis is different compared to individuals without periodontal disease. For this reason, it is important to investigate the immune function represented by phagocytosis in periodontitis to gain insight into the defense against pathogens involved in disease conditions and the pathophysiological mechanism involved, including those of infectious nature, which are present during the course of the disease. Therefore, this study evaluated the phagocytic function of monocytes and neutrophils in periodontal disease, in comparison with control individuals without periodontal disease.

\section{MATERIAL AND METHODS}

\section{Subjects and study groups}

This study was approved by the Institutional Review Board of the Health Sciences Faculty - UnB University of Brasilia (045/2008). The subjects were evaluated and selected for inclusion in the present study at the periodontal clinic of the University Hospital of Brasilia, Brazil. The sample included 30 subjects with periodontal disease and 27 subjects with healthy periodontium, all otherwise healthy and non-smokers. The periodontitis group consisted of 20 women and 10 men (age range $21-45$ years, mean age 34.5 years) with at least 18 present teeth. The severe periodontitis was diagnosed according to the following inclusion criteria ${ }^{23}$ : radiographic evidence of bone loss extending to $\geq 30 \%$ of the root length in multiple teeth, age $\geq 18$ years, presence of $\geq 2$ teeth/quadrant with a pocket depth of $\geq 6 \mathrm{~mm}$ and concomitant attachment loss of $>3 \mathrm{~mm}$. The control group consisted of 18 women and 9 men (age range 21-44 years, mean age 34 years) with clinical probing depths (PD) $\leq 3 \mathrm{~mm}$ and clinical attachment level $(C A L) \leq 3 \mathrm{~mm}, \leq 10 \%$ sites with bleeding on probing and no radiographic evidence of bone loss. The following exclusion criteria were considered: previous mechanical periodontal therapy and antimicrobial therapy for systemic or topical oral use in the last 12 months, pregnant or lactating women, diabetes, morbid obesity, autoimmune, infectious, allergic, and gastrointestinal diseases, malnutrition, renal alterations, cancer or any other clinical situation that might alter the function of the immune system, use of medications that could alter the level of inflammatory mediators, and smoking.

\section{Clinical examination}

The initial periodontal evaluation of each patient included periapical radiographic documentation by the parallelism technique. The clinical examinations were performed by an experienced examiner and included visible plaque accumulation (PI) without the use of any disclosing agent, bleeding on probing $(B O P)$, probing depth (PD) and clinical attachment level (CAL). The measurements were assessed at four sites around each tooth, namely buccal, lingual and proximal sites (the greatest depth was recorded for each proximal surface) using a manual probe (Michigan O probe with Williams markings), excluding third molars. Analyses of degrees of mobility and furcation involvement were recorded.

\section{Phagocytosis test}

Phagocytosis of Saccharomyces cerevisiae was adapted from a technique previously described ${ }^{19}$. Briefly, samples of $40 \mu \mathrm{L}$ per marked area of heparinized whole peripheral blood obtained by venipuncture from each subject were placed on clean glass slides containing 8 marked areas with 7-mm diameter each, in duplicate preparations, and incubated in a wet chamber for $45 \mathrm{~min}$ at $37^{\circ} \mathrm{C}$. The slides were then rinsed with $0.15 \mathrm{M}$ phosphate-buffered saline (PBS) $\mathrm{pH} 7.2$ at $37^{\circ} \mathrm{C}$ to remove non-adherent cells. After washing, neutrophils and monocytes remained adhered onto the slide approximately in the same proportion as they were in the whole blood. Adherent cells $(12,534 \pm 5,050$ cells/marked area; 5.63 $\pm 0.85 \%$ monocytes and $93.5 \pm 1.08 \%$ neutrophils) were incubated with a suspension of $2.5 \times 10^{5} \mathrm{~S}$. cerevisiae in $20 \mu \mathrm{L}$ Hanks-tris (Sigma Co., St Louis, MO, USA) $\mathrm{pH} 7.2$, with $10 \%$ heat-inactivated fetal calf serum (FCS) (Gibco/Invitrogen, Grand Island, NY, USA) for $30 \mathrm{~min}$ in a wet chamber at $37^{\circ} \mathrm{C}$. To evaluate the influence of complement molecules on phagocytosis in periodontitis the $S$. cerevisiae were incubated at $37^{\circ} \mathrm{C}$ for 30 min with $10 \%$ fresh serum from the donor in Hanks-Tris solution. Slides were then rinsed with $0.15 \mathrm{M}$ PBS at $37^{\circ} \mathrm{C}$ to eliminate nonphagocytosed $S$. cerevisiae and the final washing was done with $30 \%$ FCS in Hanks-tris. The slides were fixed with absolute methanol and stained with $10 \%$ Giemsa solution. The number of S. cerevisiae phagocytozed by 200 monocytes or by 200 neutrophils in individual preparations was 
assessed by light microscopy. Microscopic fields distributed throughout the slide were randomly selected and all monocytes or neutrophils in each particular field were examined. The PhI was calculated as the mean number of phagocytozed S. cerevisiae per phagocytosing monocytes or neutrophils, multiplied by the percentage of these cells engaged in phagocytosis ${ }^{19}$.

Baking yeast (S. cerevisiae) was prepared according to a technique previously described ${ }^{20}$.

\section{Statistical analysis}

Statistical analysis was performed using the Prism ${ }^{\circledR}$ software, 2005 (Graphpad, San Diego, CA, USA). Beforehand the variables in the samples were previously verified for normality, using the Skewness and Kurtosis and Kolmogorov-Smirnov tests. The t-test was used for comparison between two variables with normal distribution, and the Mann-Whitney test was used for those that did not present normal distribution. The differences between variables were considered statistically significant when the bi-caudal probability of their occurrence due to chance (error type I) was lower than $5 \%(p<0.05)$. As several data showed nonnormal distribution, for homogeneity, all data were graphically expressed as median, quartiles and extremes.

\section{RESULTS}

\section{Clinical and demographic characteristics}

The clinical and demographic characteristics of the two groups are summarized in Table 1. Distribution of age and gender was similar between control and periodontitis groups. Subjects with periodontitis had significantly higher body mass index than control $(p=0.001)$. Statistically significant differences were also observed for all periodontal parameters. Subjects with periodontitis showed severe destructive periodontal disease when observed by the percentage of sites with PD $\leq 4 \mathrm{~mm}$ to $\geq 7 \mathrm{~mm}$ and $\mathrm{CAL} \leq 4 \mathrm{~mm}$ to $\geq 7 \mathrm{~mm}$. The hematological characteristics of the groups are listed in Table 2. No statistically significant difference was observed between groups, except for $C$ reactive protein (CRP). The mean of serum levels of CRP was $0.21 \pm 0.25$ for the control group and $0.51 \pm 0.62$ for the periodontitis group $(p=0.01)$.

\section{Phagocytosis test}

PhI of neutrophils and monocytes for nonopsonized S. cerevisiae

The median of the PhI of neutrophils from individuals with periodontitis (1.5) was significantly lower than that of normal controls (3.0), $(p=0.01$, Mann-Whitney test) (Figure 1A). This occurred

Table 1- Demographic characteristics and full-mouth clinical parameters

\begin{tabular}{|c|c|c|c|}
\hline $\begin{array}{c}\text { Characteristics/ } \\
\text { Parameters }\end{array}$ & $\begin{array}{l}\text { Control } \\
(n=27)\end{array}$ & $\begin{array}{l}\text { Periodontitis } \\
\qquad(n=30)\end{array}$ & (p) Test \\
\hline Age (years; mean+SD) & $33.2 \pm 6.4$ & $33.5 \pm 6.8$ & $0.8441^{* *}$ \\
\hline Gender (males/females: $n$ ) & $9 / 18$ & $10 / 20$ & $1.0000^{* * *}$ \\
\hline Numbers of teeth (mean+SD) & $28 \pm 1.3$ & $27.5 \pm 4.8$ & $0.3545^{*}$ \\
\hline BMI & $20.3 \pm 1.28$ & $26.53 \pm 5.49$ & $0.0001^{*}$ \\
\hline $\mathrm{SBP}(\mathrm{mmHg})$ & $120.9 \pm 4.8$ & $122.4 \pm 14.8$ & $0.9188^{*}$ \\
\hline $\mathrm{DBP}(\mathrm{mmHg})$ & $80.5 \pm 2.5$ & $80.9 \pm 11.6$ & $0.0744^{*}$ \\
\hline $\mathrm{PI}(\% ;$ mean+SD) & $4.8 \pm 2.1$ & $61.37 \pm 33.59$ & $0.0001^{*}$ \\
\hline BOP $(\% ;$ mean+SD) & $2.7 \pm 1.2$ & $41.83 \pm 30.04$ & $0.0001^{*}$ \\
\hline \multicolumn{4}{|l|}{ PD (mm; mean+SD) } \\
\hline$\leq 4 \mathrm{~mm}$ & $100 \%$ & $3.8 \pm 3.97$ & \\
\hline $5-6 \mathrm{~mm}$ & $0 \%$ & $16.1 \pm 9.33$ & \\
\hline$\geq 7 \mathrm{~mm}$ & $0 \%$ & $9.8 \pm 8.8$ & \\
\hline \multicolumn{4}{|l|}{ CAL (mm; mean+SD) } \\
\hline$\leq 4 \mathrm{~mm}$ & $100 \%$ & $5.8 \pm 4.5$ & \\
\hline $5-6 \mathrm{~mm}$ & $0 \%$ & $17.9 \pm 9.2$ & \\
\hline$\geq 7 \mathrm{~mm}$ & $0 \%$ & $13.0 \pm 11.4$ & \\
\hline
\end{tabular}

* Mann-Whitney test

** t-test

${ }^{* * *}$ Chi-Square test, BMI (body mass index), SBP (Systolic Blood Pressure), DBP (Diastolic Blood Pressure)

PI (plaque index), BOP (bleeding on probing), PD (probing depth), CAL (clinical attachment level). 
Table 2- Biochemical and hematological characteristics

\begin{tabular}{lccc}
\hline \multicolumn{1}{c}{$\begin{array}{c}\text { Characteristics/ } \\
\text { Parameters }\end{array}$} & $\begin{array}{c}\text { Control } \\
(\mathbf{n = 2 7})\end{array}$ & $\begin{array}{c}\text { Periodontitis } \\
(\mathbf{n = 3 0})\end{array}$ & (p) Test \\
\hline Triglycerides $(\mathrm{mg} / \mathrm{dL})$ & $86.1 \pm 35.4$ & $98.9 \pm 46.2$ & $0.2675^{*}$ \\
\hline Total cholesterol (mg/dL) & $172 \pm 31.4$ & $173.9 \pm 3.3$ & $0.8222^{* *}$ \\
\hline HDL-cholesterol (mg/dL) & $48.2 \pm 12$ & $42.5 \pm 12.3$ & $0.0747^{*}$ \\
\hline LDL-cholesterol (mg/dL) & $106 \pm 24.4$ & $111.5 \pm 27.3$ & $0.4348^{* *}$ \\
\hline Glucose (mg/dL) & $85.3 \pm 6.7$ & $90.6 \pm 14.1$ & $0.1528^{*}$ \\
Eosinophils & $146.1 \pm 103.3$ & $222.9 \pm 175.8$ & $0.0646^{*}$ \\
Basophils & $14.7 \pm 31.8$ & $8.1 \pm 21.3$ & $0.627^{*}$ \\
Lymphocytes & $2,239 \pm 530.7$ & $2,137 \pm 499.5$ & $0.4607^{* *}$ \\
Monocytes & $422.6 \pm 140.9$ & $388.5 \pm 160.1$ & $0.2369^{*}$ \\
Total leukocytes & $6,093 \pm 1,216$ & $6,308 \pm 1.473$ & $0.5111^{*}$ \\
C-Reactive Protein & $0.21 \pm 0.25$ & $0.51 \pm 0.62$ & $0.0105^{*}$ \\
\hline
\end{tabular}

* Mann-Whitney test

** t-test

because there was a reduction in the percentage of neutrophils involved in phagocytosis in the periodontitis group $(0.50 \%)$ when compared to the normal control group $(2.25 \%)(p=0.006$, MannWhitney test) (Figure $1 \mathrm{C})$, whereas no statistically significant difference was observed between groups for the mean number of yeasts adhered to/ingested by neutrophils ( $p=0.50$, Mann-Whitney test) (Figure 1B).

The same situation was verified when phagocytosis by monocytes was tested. The median of the PhI of monocytes from individuals with periodontitis (13.23) was significantly lower than that of the normal controls (26.13) $(p=0.02$, MannWhitney test) (Figure 1D), because a statistically significant reduction in the percentage of monocytes involved in phagocytosis was observed in the periodontitis group $(8.50 \%)$ when compared to the control group (17.25\%) $(p=0.01$, Mann Whitney test) (Figure 1F). Conversely, no difference was observed between groups for the mean number of yeasts adhered to/ingested by monocytes $(p=0.71$, t test) (Figure 1E).

PhI of neutrophils and monocytes for opsonized S. cerevisiae

A different situation occurred when phagocytosis was evaluated using sensitized $S$. cerevisae. No statistical difference was observed in the $\mathrm{PhI}$ of neutrophils between individuals with periodontitis (117.9) and normal control individuals (157.0) $(p=0.14$, t-test) (Figure $2 A)$. Periodontitis had no influence on the mean number of yeasts adhered/ingested by neutrophils ( $p=0.15$, MannWhitney test) as illustrated in Figure 2B. There was no statistical difference in the involvement of neutrophils in phagocytosis between the two study groups ( $p=0.16$, Mann-Whitney test) (Figure 2C).
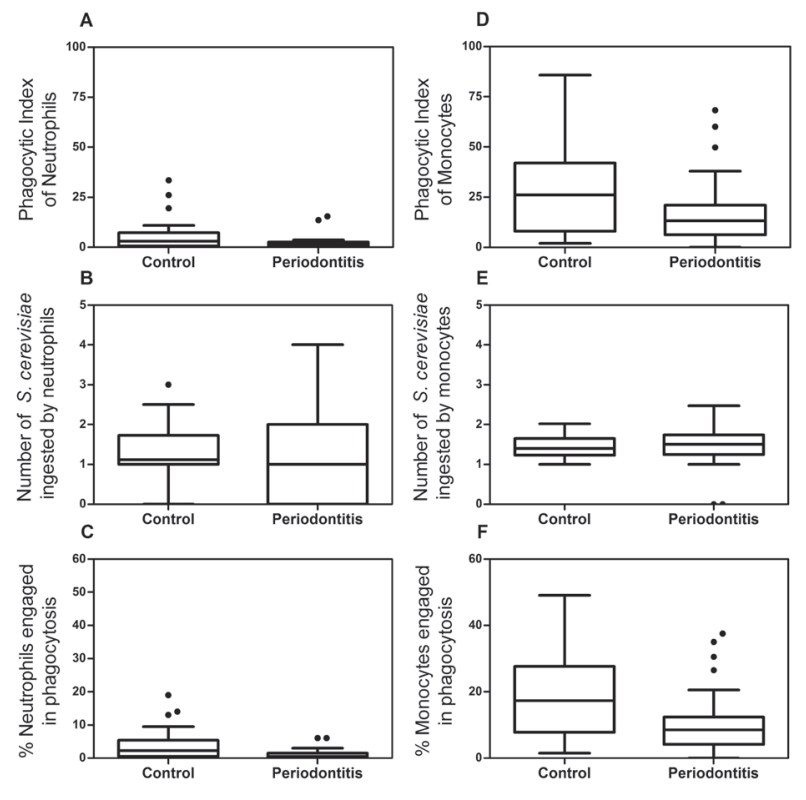

Figure 1- In vitro evaluation of the phagocytic capacity of neutrophils (left) or monocytes (right) in individuals with periodontal disease $(P)$ and normal control individuals, using $2.5 \times 10^{5}$ non sensitized yeast per well. A: Reduction of the phagocytic index $(p=0.01$, Mann-Whitney test). $B$ : Mean number of yeasts adhered to/ingested by neutrophils ( $p=0.50$, Mann-Whitney test). C: Reduction of the percent of neutrophils involved in phagocytosis $(p=0.006$, Mann-Whitney test). $D$ : Reduction of the phagocytic index of monocytes ( $p=0.0,2$, Mann-Whitney test). $E$ : Mean number of yeasts adhered to/ingested by monocytes ( $p=0.71$, t-test). $F$ : Reduction in the percentage of monocytes involved in phagocytosis $(p=0.01$, MannWhitney test). Data were expressed as median, quartile and extreme. Outlier values are marked 

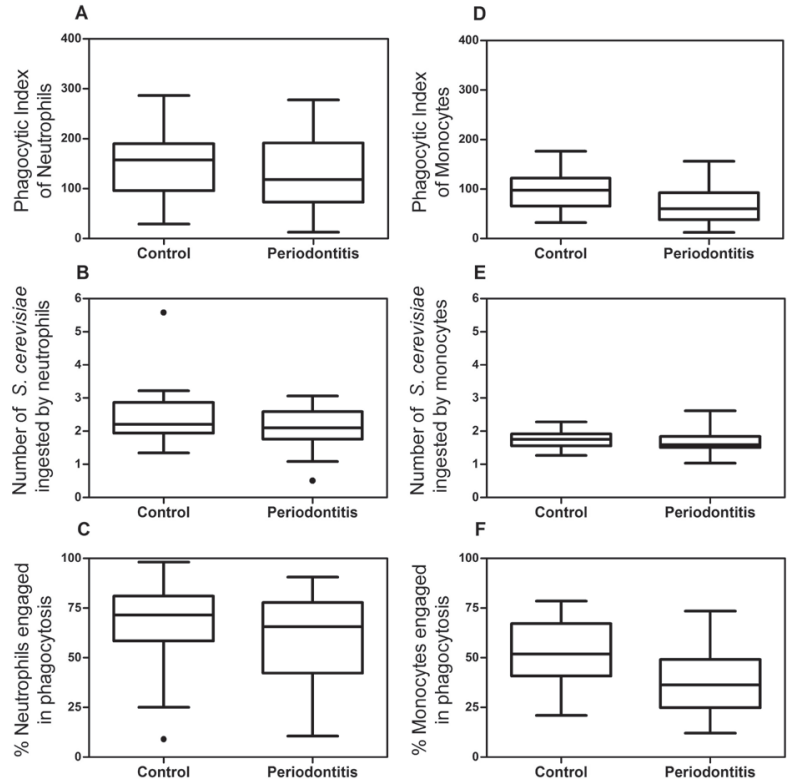

Figure 2- In vitro evaluation of the phagocytic capacity of neutrophils (left) or monocytes (right) in individuals with periodontal disease $(P)$ and normal control individuals, using $2.5 \times 10^{5}$ sensitized yeast per well. A: Phagocytic Index $(p=0.14$, $t-$ test $)$. B: Mean number of yeasts adhered to/ingested by neutrophils $(p=0.15$ MannWhitney test). C: Percentage of neutrophils involved in phagocytosis ( $p=0.16$, Mann-Whitney test). $D$ : Reduction of the Phagocytic Index of monocytes ( $p=0.005$, t- test). $\mathrm{E}$ : Mean number of yeasts adhered to/ingested by monocytes ( $p=0.19$, Mann-Whitney test). F: Percentage of monocytes involved in phagocytosis ( $p=0.001$, t-test). Data were expressed as median, quartile and extreme. Outlier values are marked

The median proportion of neutrophils involved in phagocytosis in the periodontitis group was $65.50 \%$, compared to $71.50 \%$ in the control group.

However, a different result was verified when phagocytosis was tested by monocytes, where the median of PhI of monocytes from individuals with periodontitis (60.10) was significantly lower than that from the normal controls (97.92) $(p=0.005$, t-test) (Figure 2D). This decrease was caused by a lower percentage of monocytes involved in phagocytosis, namely $36.25 \%$ for the periodontitis group and $51.75 \%$ for the control group ( $p=$ 0.0016 , t-test) (Figure 2F). The medians of the mean number of yeasts adhered to/ingested by monocytes were statistically similar between both groups, as illustrated in Figure 2E.

\section{DISCUSSION}

This is the first description of decreased phagocytic capacity of monocytes in human periodontal diseases. In the present study phagocytosis by monocytes and neutrophils were compared between individuals with healthy periodontium and those with periodontitis. In this work, killed Saccharomyces cerevisiae was used because receptors involved in their uptake are involved in phagocytosis by neutrophils and monocytes of pathogenic bacteria present in periodontal disease ${ }^{27}$. When using live bacteria, their virulence factors may influence phagocytosis. Thus, when investigating the phagocytosis, two lines of reasoning can be defined: the direct action of bacteria in phagocytosis and the effects of hostparasite interactions in phagocytosis. As our aim was to evaluate the effects of this interaction in the host, this justifies another stimulus provided to the cell, including yeasts. The use of different stimuli is reported in the literature: $\mathrm{N}$-formyl-L-methionylL-leucyl-L-phenylalanine (FMLP) ${ }^{29}$, Staphylococcus aureus ${ }^{5}$, Candida albicans 2,25 , Aggregatibacter actinomycetemcomitans, Porphyromonas gingivalis ${ }^{13,21}$.

The present study showed a significant reduction in the phagocytic capacity of monocytes, both using opsonized and non-opsonized S. cerevisiae (Figure $1 D$ and 2D). However, for neutrophils, the disease decreased phagocytosis only when it was assessed using non-opsonized yeasts (Figure1A). This lower phagocytic capacity of neutrophils in periodontitis has also been reported by other researchers ${ }^{2,4,12,29}$. In this study, lower phagocytosis using nonopsonized S. cerevisiae was observed in the group of individuals with periodontitis, both by neutrophils (Figure 1A) and monocytes (Figure 1D). These data suggest that the phagocytic function of both neutrophils and monocytes was intrinsically affected because, in the absence of serum components, the level of phagocytosis was lower in the periodontitis group. Regarding the monocytes, for both analyses, the $\mathrm{PhI}$ was lower in individuals with periodontal disease (Figure 1D and 2D). A reduced number of monocytes involved in phagocytosis in this group was also evident (Figure $1 \mathrm{~F}$ and $2 \mathrm{~F}$ ).

The mechanisms for the deficient response of phagocytes have not been explained yet, however some hypotheses can be suggested. It is known that many periodontal pathogens develop particular strategies for subverting the mechanisms of phagocytosis' ${ }^{27}$. Among the mechanisms of immunosuppression, the role of LPS has been considered. Studies have indicated that the LPS of $P$. gingivalis appears to be antagonists of the toll-like receptor-4, thus competing with LPS of other species to couple with that receptor. This is a possible mechanism of deficiency of the innate immune system of the host, since recognition of the bacterial pathogens identified by the toll-like receptor- 4 would be blocked ${ }^{6}$. The recruitment of neutrophils and macrophages in mice infected with $A a$ and lacking the toll-like receptor-2 led to the 
reduction in the influx of these cells in the peritoneal cavity. Infection with $A a$ in this experimental model caused a significant decrease in the cytokine and chemokine levels and reduction in the phagocytic capacity of neutrophils and monocytes, in addition to alveolar bone loss ${ }^{11}$.

Another possibility that has been described is the direct toxicity of $A a$ to neutrophils and monocytes by the production of leukotoxin ${ }^{26}$. Strains of $A a$ have various mechanisms that control phagocytosis, such as inhibition of chemotaxis and immunosuppressive and cytotoxic factors that suppress both the unspecific and specific immune responses, as well as preventing fibroblast proliferation. They also present the additional capacity to invade epithelial and endothelial cells ${ }^{15}$. Baehni, et al. ${ }^{3}$ (1979) observed by electron microscopy that the cytotoxic effects on PMN by strains of $A a$ were independent of phagocytosis. The authors suggested that soluble bacterial products may be released by bacteria. Carvalho, et al. ${ }^{4}$ (2009) found high frequency of $P$. gingivalis, Tannerella forsythia and $A a$ in individuals with aggressive periodontitis. They observed that in these individuals the frequency and quantity of $P$. gingivalis and $T$. forsythia presented negative correlation with phagocytosis by PMN. The depression of phagocytosis observed in the present study in the periodontal disease group can be justified by the action of these pathogens usually found in more severe forms of periodontal disease. In the analysis of phagocytosis by neutrophils using yeasts sensitized with fresh serum from the individual, although the PhI in individuals from the periodontitis group presented a trend toward reduction, the results did not differ statistically from those of the control group (Figure 2A). This suggests that there was no considerable change in complement an immunoglobulin receptors on neutrophils, as well as considerable influence of other serological factors in this group.

Different from the present results, studies in individuals with more severe forms of periodontal disease demonstrate that regulatory factors in serum may modulate functions of PMNs. Depression of the chemotactic response in patients with localized aggressive periodontitis may not be an abnormality associated with the cell, but rather a consequence of the elevation of the serum concentration of cytokines produced during the host-parasite interaction. The TNF-alpha and IL-1 cytokines may cause a reduction in chemotactic receptors ${ }^{1}$. However, increased local production of cytokines in response to pathogens in the periodontium may result in bone loss and tissue damage typically observed in more severe forms of periodontitis ${ }^{10,30}$. Although there are no studies showing the relationship between serum levels of inflammatory mediators and phagocytosis, there may be an inverse correlation between them because depressed phagocytosis is always found in periodontal pockets ${ }^{9,24}$.

Although with small difference, the present data demonstrated that the serum level of C-reactive protein (CRP) was increased in individuals with periodontitis (Table 2). In more severe forms of periodontal disease, the association between the LPS of periodontopathogenic bacteria and inflammatory mediators has been well established ${ }^{8}$, which leads to the increase of CRP with consequent cardiovascular alterations ${ }^{18}$. Similarly, it is possible that systemic immunosuppression of phagocytic cells, as observed in this study, may reduce the defense against bacteria and fungi. It is also a possibility the fact that the absence of systemic manifestations, observed in the present study, occur due to the redundancy of immune system functions. The long-term consequences of the reduction of phagocytosis in the group with periodontitis should be further evaluated.

\section{CONCLUSIONS}

This study showed for the first time that the monocytes of peripheral blood from individuals with periodontitis present decreased phagocytosis of opsonized and non-opsonized $S$. cerevisiae in comparison with control individuals. Concerning the neutrophils, decreased phagocytosis was observed only for non-opsonized yeasts. Although the individuals did not show clinical parameters of immunodeficiency, a laboratorial decreased function of phagocytes was characterized in this work, which may be a consequence, not the cause, of periodontitis. More studies should be conducted to investigate the immune inflammatory events implicated in phagocytosis by neutrophils and monocytes in periodontitis.

\section{ACKNOWLEDGMENTS}

The authors gratefully acknowledge Mrs. Shirley Claudino Couto for the excellent technical assistance and Dr. Raquel de Menezes Barbosa and Dr. Mariah Bastos Braun for helping with the phagocytosis test evaluation. The authors report no conflicts of interest related to this study. 


\section{REFERENCES}

1- Agarwal S, Suzuki JB, Riccelli AE. Role of cytokines in the modulation of neutrophil chemotaxis in localized juvenile periodontitis. J Periodontal Res. 1994;29:127-37.

2- Asif K, Kothiwale SV. Phagocytic activity of peripheral blood and crevicular phagocytes in health and periodontal disease. J Indian Soc Periodontol. 2010;14:8-11.

3- Baehni P, Tsai CC, McArthur WP, Hammond BF, Taichman NS. Interaction of inflammatory cells and oral microorganisms. VIII. Detection of leukotoxic activity of a plaque-derived gram-negative microorganism. Infect Immun. 1979;24:233-43.

4- Carvalho RP, Mesquita JS, Bonomo A, Elsas PX, Colombo AP. Relationship of neutrophil phagocytosis and oxidative burst with the subgingival microbiota of generalized agressive periodontitis. Oral Microbiol Immunol. 2009;24:124-32.

5- Cortelli JR, Roman-Torres CV, Aquino DR, Franco GC, Costa FO, Cortelli SC. Occurrence of Aggregatibacter actinomycetemcomitans in Brazilians with chronic periodontitis. Braz Oral Res. 2010;24:21723.

6- Darveau RP, Pham TT, Lemley K, Reife RA, Bainbridge BW, Coats $\mathrm{SR}$, et al. Porphyromonas gingivalis lipopolysaccharide contains multiple lipid A species that functionally interact with both toll-like receptors 2 and 4. Infect Immun. 2004;72:5041-51.

7- Deas DE, Mackey SA, McDonnell HT. Systemic disease and periodontitis: manifestations of neutrophil dysfunction. Periodontol 2000. 2003;23:82-104.

8- Duarte PM, Rocha M, Sampaio E, Mestnik MJ, Feres M, Figueiredo LC, et al. Serum levels of cytokines in subjects with generalized chronic and aggressive periodontitis before and after non-surgical periodontal therapy: a pilot study. J Periodontol. 2010;81:1056-63.

9- Eick S, Pfister W, Sigusch B, Straube E. Phagocytosis of periodontopathogenic bacteria by crevicular granulocytes is depressed in progressive periodontitis. Infection. 2000;28:301-4. 10- Figueredo CM, Ribeiro MS, Fischer RG, Gustafsson A. Increased interleukin-1beta concentration in gingival crevicular fluid as a characteristic of periodontitis. J Periodontol. 1999;70:1457-63.

11- Gelani V, Fernandes AP, Gasparoto TH, Garlet TP, Cestari TM, Lima HR, et al. The role of toll-like receptor 2 in the recognition of Aggregatibacter actinomycetemcomitans. J Periodontol. 2009;80:2010-9.

12- Gomez RS, Costa JE, Lorentz TM, Garrocho AA, NogueiraMachado JA. Chemiluminescence generation and MTT dye reduction by polymorphonuclear leukocytes from periodontal disease patients. J Periodontal Res. 1994;29:109-12.

13- Guentsch A, Puklo M, Preshaw PM, Glockmann E, Pfister W, Potempa J, et al. Neutrophils in chronic and aggressive periodontitis in interaction with Porphyromonas gingivalis and Aggregatibacter actinomycetemcomitans. J Periodontal Res. 2009;44:368-77.

14- Kantarci A, Oyaizu K, Van Dyke TE. Neutrophil-mediated tissue injury in periodontal disease pathogenesis: findings from localized aggressive periodontitis. J Periodontol. 2003;74:66-75.
15- Kurita-Ochiai T, Ochiai K, Ikeda T. Immunosuppressive effect induced by Actinobacillus actinomycetemcomitans: effect on immunoglobulin production and lymphokine synthesis. Oral Microbiol Immunol. 1992;7:338-43.

16- Lins RD, Figueiredo CR, Queiroz LM, Silveira EJ, Freitas RA. Immunohistochemical evaluation of the inflammatory response in periodontal disease. Braz Dent J. 2008;19:9-14.

17- Loss BG, Craandijk J, Hoek FJ, Wertheim-van Dillen PM, van der Velden $\mathrm{U}$. Elevation of systemic markers related to cardiovascular diseases in the peripheral blood of periodontitis patients. J Periodontol. 2000;71:1528-34.

18- Marcaccini AM, Meschiari CA, Sorgi CA, Saraiva MC, Souza AM, Faccioli LH, et al. Circulating interleukin- 6 and high-sensitivity C-reactive protein decrease after periodontal therapy in otherwise healthy subjects. J Periodontol. 2009;80:594-602.

19- Muniz-Junqueira MI, Peçanha LM, Silva-Filho VL, Cardoso MCA, Tosta CE. Novel microtechnique for assessment of postnatal maturation of the phagocytic function of neutrophils and monocytes. Clin Diagn Lab Immunol. 2003;10:1096-102.

20- Muniz-Junqueira MI, Prata A, Tosta CE. Phagocytic and bactericidal function of mouse macrophages to Salmonella typhimurium in schistosomiasis mansoni. Am J Trop Med Hyg. 1992;46:132-6.

21- Nibali L, O'Dea M, Bouma G, Parkar M, Thrasher AJ, Burns $S$, et al. Genetic variants associated with neutrophil function in aggressive periodontitis and healthy controls. J Periodontol. 2010;81:527-34.

22- Page RC, Kornman KS. The pathogenesis of human periodontitis: an introduction. Periodontol 2000. 1997;14:9-11. 23- Papapanou PN, Sedaghatfar MH, Demmer RT, Wolf DL, Yang J, Roth GA, et al. Periodontal therapy alters gene expression of peripheral blood monocytes. J Clin Periodontol. 2007;34:736-47. 24- Sigusch B, Klinger G, Holtz H, Süss J. In vitro phagocytosis by crevicular phagocytes in various forms of periodontitis. ] Periodontol. 1992;63:496-501.

25- Socransky SS, Haffajee AD, Cugini MA, Smith C, Kent RL Jr. Microbial complexes in subgingival plaque. J Clin Periodontol. $1998 ; 25: 134-44$.

26- Tsai CC, McArthur WP, Baehni PC, Hammond BF, Taichman NS. Extraction and partial characterization of a leukotoxin from a plaque-derived Gram-negative microorganism. Infect Immun. 1979;25:427-39.

27- Underhill DM, Ozinsky A. Phagocytosis of microbes: complexity in action. Annu Rev Immunol. 2002;20:825-52.

28- Van Dyke TE. The etiology and pathogenesis of periodontitis revisited [Editorial]. ] Appl Oral Sci. 2009;17:pii.

29- Van Dyke TE, Zinney W, Winkel K, Taufiq A, Offenbacher S, Arnold RR. Neutrophil function in localized juvenile periodontitis. Phagocytosis, superoxide production and specific granule release. J Periodontol. 1986;57:703-8.

30- Zhou J, Zou S, Zhao W, Zhao Y. Prostaglandin E2 level in gingival crevicular fluid and its relation to the periodontal pocket depth in patients with periodontitis. Chin Med Sci J. 1994;9:52-5. 